\title{
Representações sociais de adolescentes sobre a transmissão do HIV/AIDS nas relações sexuais: vulnerabilidades e riscos
}

\author{
Social representations of adolescents about HIV/AIDS transmission in sexual relations: vulnerabilities and \\ risks \\ Representaciones sociales de adolescentes sobre la transmisión del VIH/AIDS en relaciones sexuales: \\ vulnerabilidades y riesgos
}

Esmelsy Cabrera Garcia ${ }^{1}$ (1) Ivoneide Ribeiro $\operatorname{Costa}^{2}$ (1)

Regina Celia de Oliveira ${ }^{3}$ (1) Carlos Roberto Lyra da Silva ${ }^{4}$ (1) Amanda Regina da Silva Góis ${ }^{5}$ (C) Fátima Maria da Silva $\mathrm{Abrão}^{3}$ (C)

1. Universidade Autônoma de Santo Domingo. Santo Domingo, Santo Domingo, República Dominicana.

2. Universidade Federal de Pernambuco, Hospital das Clínicas. Recife, PE, Brasil.

3. Universidade de Pernambuco, Programa Associado de Pós-Graduação em Enfermagem. Recife, PE, Brasil.

4. Universidade Federal do Estado do Rio de Janeiro, Programa de Pós-Graduação em Enfermagem e Biociências. Rio de Janeiro, RJ, Brasil.

5. Universidade de Pernambuco. Petrolina, PE, Brasil.

Autor correspondente:

Amanda Regina da Silva Góis.

E-mail: amanda.gois@upe.br

Recebido em 29/03/2021.

Aprovado em 29/07/2021

DOI: https://doi.org/https://doi.org/10.1590/

2177-9465-EAN-2021-0083

\section{RESUMO}

Objetivo: compreender as representações sociais de adolescentes sobre vulnerabilidades e riscos para contrair o HIV/Aids nas relações sexuais. Método: estudo de representações sociais, realizado a partir de entrevistas individuais com 15 adolescentes e grupo focal com oito adolescentes de uma escola pública do Recife, Pernambuco, Brasil. Os dados foram coletados no período de maio a outubro de 2016. Para tratamento dos dados, empregou-se a técnica de análise de conteúdo temática-categoria com o auxílio do Software QSR Nvivo11. Resultados: as categorias: riscos de contrair HIV/Aids nas relações sexuais; e dos riscos de contrair HIV/Aids nas relações sexuais às vulnerabilidades abrigam os temas não usar preservativo, ter usado álcoo e/ou drogas, ter vários parceiros, ter dificuldades de acesso aos serviços de saúde e de educação sexual nas escolas; e não ter conversas com os pais ou familiares. Conclusão e implicações para a prática: compreendeu-se que as representações sociais circulam no imaginário e no ambiente escolar, interferindo na realidade dos adolescentes. Propõe-se priorizar políticas públicas focadas nas dimensões emocionais, afetivas e sociais.

Palavras-chave: Percepção Social; Adolescente; Saúde Sexual; HIV; Enfermagem.

\section{ABSTRACT}

Objective: To understand social representations of adolescents about vulnerabilities and risks of contracting HIV/AIDS in sexual relations. Method: Study of social representations, based on individual interviews with 15 adolescents and a focus group with eight adolescents from a public school in Recife, Pernambuco, Brazil. Data were collected from May to October 2016. For the processing of data, the thematic-categorial content analysis technique was used with the aid of the QSR Nvivo11 Software. Results: The categories: risks of contracting HIV/AIDS in sexual relations; and from the risks of contracting HIV/AIDS in sexual relations to the vulnerabilities cover the themes of not using condoms, having used alcohol and/or drugs, having several partners having difficulties in accessing health and sex education services in schools; and not having conversations with parents or family members. Conclusion and implications for practice: it was understood that social representations circulate in the imaginary and in the school environment, interfering in the reality of adolescents. It is proposed to prioritize public policies focused on the emotional, affective and social dimensions.

Keywords: Social Perception; Adolescent; Sexual Health; HIV; Nursing.

\section{RESUMEN}

Objetivo: comprender las representaciones sociales de adolescentes acerca de las vulnerabilidades y riesgos de contraer e VIH/SIDA en relaciones sexuales. Método: estudio de representaciones sociales realizado a partir de entrevistas individuales con 15 adolescentes y un grupo focal con ocho adolescentes de una escuela pública en Recife, Pernambuco, Brasil. Los datos se recolectaron entre mayo y octubre de 2016. Para el tratamiento de los datos, se utilizó la técnica de análisis de contenido temático-categórico, con la ayuda del software QSR Nvivo11. Resultados: las categorías riesgos de contraer el VIH/SIDA en las relaciones sexuales; $y$ de los riesgos de contraer el VIH/SIDA en las relaciones sexuales a las vulnerabilidades que cubren los temas de no usar condón, haber consumido alcohol y/o drogas, tener varios pares, tener dificultades para acceder a los servicios de salud y de educación sexual en las escuelas; y no tener conversaciones con los padres o miembros de la familia. Conclusión e implicaciones para la práctica: se entendió que las representaciones sociales circulan en el imaginario y en el ámbito escolar, interfiriendo en la realidad de los adolescentes. Se propone priorizar políticas públicas enfocadas en las dimensiones emocionales, afectivas y sociales.

Palabras clave: Percepción Social; Adolescente; Salud Sexual; VIH; Enfermería. 


\section{INTRODUÇÃO}

O número de novos casos do vírus da imunodeficiência humana (HIV) continua aumentando entre adolescentes ao longo dos anos. No Brasil, somente em 2018, $21 \%$ de todos os novos diagnósticos de HIV ocorreram em adolescentes, destes $87 \%$ eram homens na faixa etária de 13 a 19 anos, e a principal via de transmissão a sexual ${ }^{1}$.

Nesse sentido, há uma necessidade urgente de tornar os adolescentes capazes de reconhecer as chances que possuem para contrair o HIV/Aids e se proteger dele e de outras infecções sexualmente transmissíveis (IST).

Para aprofundar a compreensão e contribuir para esse tema, adotou-se, neste estudo, a definição de vulnerabilidade que remete a complexidade das relações intersubjetivas e os contextos sociais e políticos relacionados às situações sistemáticas de desrespeito aos diretos que levam a maior exposição a danos à saúde, considerando a capacidade de reconhecimento e resposta social diante dos agravos de saúde das pessoas². Essa definição será discutida em contraponto aos riscos, que consideram apenas a probabilidade ou as chances que um adolescente possui de contrair o vírus em relação a população de outras faixas etárias.

Nesse sentido, entende-se que a superação de riscos e vulnerabilidades envolvem, a garantia de direito a um desenvolvimento sexual seguro e saudável, através da implementação de estratégias de promoção da saúde e programas de prevenção que busquem melhorar os índices de deteção precoce dos casos, uma vez que a população adolescente não se reconhece como vulnerável, mesmo após o sexo sem proteção, e que o início da atividade sexual é cada vez mais precoce. Essas estratégias e programas devem considerar não apenas riscos, mas as vulnerabilidades individuais, sociais e institucionais para adolescentes ${ }^{3-5}$.

Sobre os programas de prevenção destaca-se, como interesse relacionado ao objeto de estudo, o Programa Saúde na Escola (PSE). Desenvolvido no âmbito da política intersetorial pelo Ministério da Saúde e da Educação em conformidade com as estratégias prioritárias da Política Nacional de Promoção da Saúde (PNPS), as ações educativas são executadas pelas equipes de Saúde da Família e pelos profissionais das escolas com foco na saúde de crianças e adolescentes ${ }^{6}$.

Nesse sentido, parte-se do pressuposto de que compreender como pensam os adolescentes dentro da sua realidade, a escola possibilita a promoção da saúde, visto que alguns conhecimentos de senso comum podem influenciar mudanças de atitudes para a prevenção. Desse modo, pesquisas que adotam a Teoria das Representações Sociais (TRS) como um referencial teoricometodológico, ${ }^{7}$ podem contribuir para esses fins.

O presente estudo teve como objetivo compreender as representações sociais de adolescentes sobre vulnerabilidades e riscos para contrair o HIV/Aids nas relações sexuais.

\section{MÉTODO}

Estudo qualitativo, exploratório e descritivo orientado pela Teoria das Representações Sociais (TRS) com abordagem processual, que parte da definição das representações sociais como uma forma de conhecimento do senso comum, compartilhado e elaborado socialmente, que permite a construção de uma realidade comum aos indivíduos pertencentes a um grupo social ${ }^{7}$.

Realizou-se a pesquisa em uma escola pública de referência para o ensino médio em período integral, no Município do Recife, Pernambuco, Brasil selecionada por integrar-se ao Programa Saúde na Escola (PSE) e às ações de um projeto de extensão realizado pelo Grupo de Estudos e Pesquisa em Epistemologia e Fundamentos do Cuidar na Saúde e Enfermagem da Universidade de Pernambuco (UPE), Faculdade de Enfermagem Nossa Senhora das Graças (FENSG).

Os critérios de inclusão foram estar regularmente matriculado no ensino médio e ser adolescente. Considerou-se, neste estudo, adolescentes aqueles da faixa etária de 12 a 18 anos de idade, como estabelece o Estatuto da Criança e do Adolescente (ECA), Lei 8.069, de $1990 .^{8}$ Os critérios de exclusão consistem em ser aluno que não possui horário livre nas atividades escolares para participar da pesquisa. Destaca-se que participaram do estudo adolescentes de ambos os sexos, com idade entre 17 e 18 anos, em virtude da maior disponibilidade de horário nas turmas de segundo e terceiro ano do ensino médio, faixa etária prevista dessas séries.

Quanto a operacionalização da coleta de dados, iniciouse no mês de maio e foi concluída em outubro de 2016, após uma aproximação com a coordenadora pedagógica da escola para entrada no campo e indicação dos momentos para que a equipe de pesquisa pudesse fazer os convites aos estudantes das duas turmas do primeiro e segundo ano e uma turma do terceiro ano do ensino médio.

$\mathrm{Na}$ etapa inicial da pesquisa, após o convite e emprego do Termo de Assentimento Livre e Esclarecido (TACLE) que foi assinado pelos adolescentes e do Termo de Consentimento Livre e Esclarecido (TCLE) pelos responsáveis, quando menores de 18 anos, procedeu-se a técnica de entrevista individual com 15 estudantes, com objetivo de acessar às informações necessárias para descrever o perfil sociodemográfico dos participantes do estudo, como idade, sexo, etnia ou raça, local de residência, com quem mora, condição laboral e questões elaboradas pelas pesquisadoras relacionadas ao tema da pesquisa: conte o que você sabe sobre o HIV? Você pode nos contar como se transmite HIV? Como você teve informações sobre o HIV? As entrevistas foram conduzidas nos meses de junho a agosto de 2016 por uma mestranda em sala reservada com duração média de 22 minutos.

$\mathrm{Na}$ etapa seguinte, objetivou-se aprofundar a temática e, para isso, utilizou-se da técnica de grupo focal, dessa vez, dos 15 estudantes, apenas oito continuaram disponíveis para participar do estudo. Os dados foram coletados até a saturação, ou seja, até alcançar os objetivos propostos ${ }^{9-10}$. Realizou-se três encontros dos meses de setembro a outubro de 2016, com uma média de duração de uma hora e quarenta minutos para cada encontro, organizados em função das proposições incentivadoras: fale sobre o que você entende sobre os riscos de contrair uma doença através do sexo (relações sexuais); fale sobre o que você 
sabe sobre HIV e Aids; e da pergunta norteadora: quais são os riscos de contrair HIV e Aids nas relações sexuais?

Estiveram presentes nos encontros, além dos adolescentes, duas mestrandas em enfermagem e uma mestranda em hebiatria. Para favorecer a conexão com os adolescentes, e incentivar a participação, realizou-se diferentes dinâmicas de grupos e recursos lúdicos como a elaboração de parodias às músicas "Brega", gênero popular local. Muitas dessas músicas são criticadas pelo conteúdo sexual e erótico, no entanto são apreciadas pelos adolescentes da região. Todos os encontros aconteceram em uma sala privada disponibilizada pela escola.

As entrevistas e reuniões foram gravadas em formato digital e, posteriormente, transcritas na íntegra, contudo não fo solicitado aos participantes conferência dos textos, para que não houvesse comprometimentos nas participações nos encontros seguintes, por vergonha ou inibição. Para garantir o anonimato, optou-se por utilizar a letra "E", para referir-se a "estudante", seguida do número correspondente à sequência de realização das entrevistas e grupos focais.

A análise dos dados foi realizada com o suporte do Software QSR Nvivo11, utilizando-se a técnica de análise de conteúdo temático-categorial ${ }^{7}$. Na primeira etapa, denominada pré-análise, realizou-se a preparação do corpus seguindo as especificações do software. Na etapa seguinte, a exploração do material realizou-se formação das unidades de significado, identificandose os elementos intermediários de análise e a criação de nós para alocação dos trechos analisados. Emergiram categorias e temas de modo a permitir a reorganização do pensamento social acerca das representações sociais dos adolescentes sobre vulnerabilidades e riscos para contrair HIV/Aids nas relações sexuais interpretados à luz da TRS e da literatura pertinente, caracterizando a última etapa, tratamento dos resultados ${ }^{9-11}$.

O projeto foi submetido ao Comitê de ética em pesquisa da Universidade de Pernambuco CAAE: 53907716.7.0000.5192, e após obter o parecer favorável em 09 de maio de 2016, com o № 1.535 .999 a coleta foi iniciada.

\section{RESULTADOS}

Para conhecer o contexto de vida desses adolescentes e subsidiar as compreensões das representações sociais dos mesmos acerca das vulnerabilidades e riscos para contrair o HIV/Aids nas relações sexuais, realizou-se a caracterização dos participantes e identificou-se que possuem idade entre 17 e 18 anos, sendo que $13(93,4 \%)$ possuíam 18 anos, nove (60\%) eram do sexo masculino. Quanto a cor ou etnia dos participantes houve um maior número de declarados pardos, totalizando $12(80,2 \%)$ adolescentes.

Uma alta porcentagem dos adolescentes mora na periferia da cidade do Recife 11 (73,3\%). No questionamento referente a com quem o adolescente reside apenas cinco $(33,4 \%)$ moravam com ambos os pais. Relacionado a atividades de trabalho um $(6,6 \%)$ trabalhava, ajudando a mãe em seu respectivo emprego.

Quando questionados sobre como se transmite o vírus 14 (93,4\%) responderam que por meio das relações sexuais ou do compartilhamento de seringas e agulhas, um (6,6\%) respondeu que no beijo na boca e relações sexuais. No que se refere a cura 14 (93,4\%) concordaram que ainda não existe uma cura para o HIV/Aids.

Quanto aos dados analisados com o uso do software Nvivo 11, foram identificadas 513 unidades de registro (UR), distribuídas em duas categorias com três temas cada, apresentadas a seguir.

\section{Riscos de contrair HIV/Aids nas relações sexuais}

A categoria engloba as verbalizações referentes aos riscos de contrair HIV/Aids nas relações sexuais, que para os adolescentes que participaram do estudo compreendem: não usar preservativo, ter usado álcool e/ou drogas, e ter relações sexuais com vários parceiros. Como apresenta a Figura 1.

Para os adolescentes, o risco de contrair HIV nas relações sexuais está relacionado a não usar preservativo, e quanto

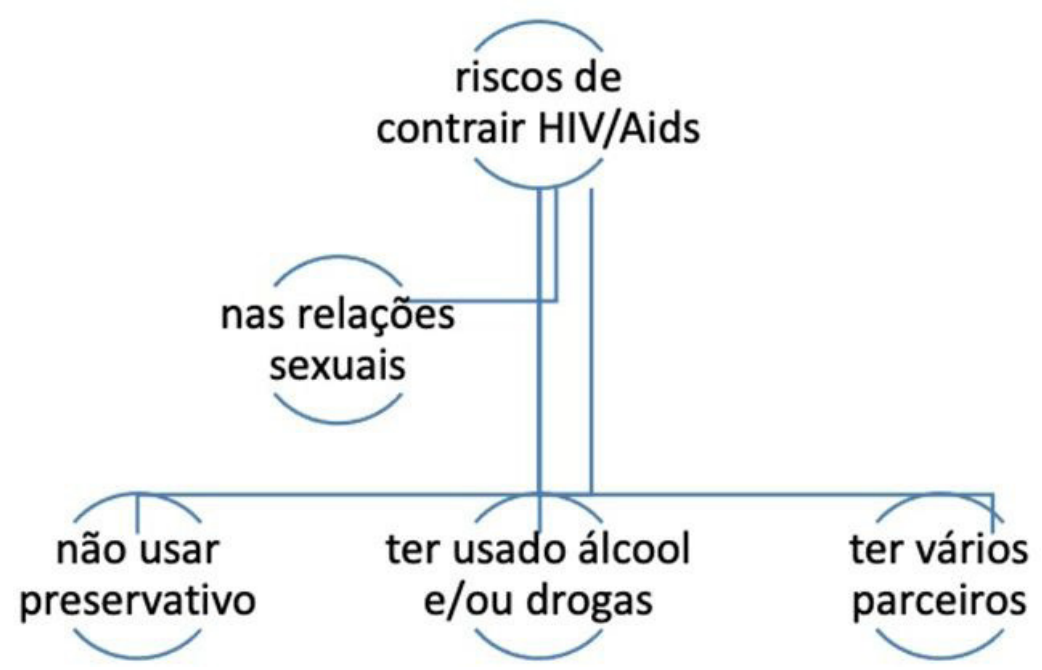

Figura 1. Organograma da categoria riscos de contrair HIV/Aids nas relações sexuais. Recife, PE, Brasil, 2017. Fonte: Pesquisadores 
aos motivos para o não uso foi possível compreender que envolvem às questões de gênero como o prazer sexual para os adolescentes masculinos e as dificuldades femininas para levar consigo o preservativo.

[...] se não andar com preservativo.... você tem que se policiar.... (E1-grupo focal)

Uma pessoa que sai com camisinha... uma de tantas... porque a pessoa vai para a balada ... e a maioria ali.... vão para pegargeral ((gíria relacionada a busca por parceiros sexuais)) principalmente... os homens [...]os homens, muitas vezes, dão uma de esquecidos... dizem não... porque o sexo sem camisinha é melhor... são mais prazerosos.... que isso que aquilo.... mulher não vai levar...nunca ouvi dizer que em uma balada... mulher levou camisinha até porque tem o tabu da camisinha feminina [...] (E2 - grupo focal).

Das falas dos adolescentes entende-se que mesmo sabendo quais são os fatores de risco para contrair HIV por meio das relações sexuais, eles se expõem e expressam que não se protegem por diferentes fatores psicoemocionais e sociais. Entre esses fatores, encontrou-se um, que seria muito importante para ter relações sexuais sem preservativo, mesmo diante do conhecimento do risco que esse ato representa para transmissão do HIV, é a confiança que depositam no parceiro ou o conceito de fidelidade e, por isso, não utilizam o preservativo como demonstração desse sentimento relacional amoroso ou romântico.

Tem que ter confiança.... para ter relações sexuais... ou porque gosta da pessoa... só isso [...] (E4 - grupo focal).

[...]se for com a minha namorada eu não ligo ((para o uso de preservativo)) [...] porque já namoramos[...] temos um relacionamento fechado...(E7-entrevista individual)

Os participantes do estudo consideram que a doença pode ser um resultado da infidelidade entre casais.

[...]é casada com esposo direitinho... mas não se satisfaz com o que tem e vai procurar fora e acaba adquirindo uma doença e passa para dentro de casa... para a esposa ou esposo e isso vai dispersando. (E8-grupo focal).

[...] ela dizia isso ((com relação ao uso de preservativo)) dizia que você pode achar que seu parceiro pode estar sendo fiel... mas ele pode não estar sendo fiel e você pode pegar uma doença.... por causa dele... (E5-grupo focal).

Outro motivo, para além do romântico foi a pressa para consolidar o ato sexual. Nessa abordagem, os adolescentes oferecem maior prioridade em ter relações sexuais sem pensar nas consequências depois do ato consumado, já que a intensidade do momento é para eles maior do que os resultados que podem gerar, como expressam as falas.
[...] mas têm pessoas que na hora $H$ ((da relação sexual)) não pensam direito [...] não dá para pensar em parar [...] nem dá para pensar em (palavra de baixo calão) de proteção nenhum. (E4-grupo focal).

Tem meninas que você fica tentando transar e elas não querem... então, quando você consegue não vai parar para pensar na (palavra de baixo calão) da camisinha. (E6-grupo focal).

Na mesma categoria, o tema ter relações sexuais com vários parceiros ou alteração frequente de parceiros sexuais é identificado pelos adolescentes como risco para contrair HIV/Aids, como expressam nas falas seguintes, enquanto a contradição de possuir um único parceiro também se revelou como um risco, quando não há o uso do preservativo, como revelou o tema anterior.

[...] tendo relações com pessoas que você não conhece[...] que você não sabe o histórico de saúde sexual dela ou dele para você ter relações sexuais [...]ficando com vários caras ((gíria para rapazes ou homens)) em uma noite.... ou como com várias meninas em uma noite[...] saibam que eu não faço isso.... (risadas)... (E4-grupo focal).

[...] é ficando com vários caras ((gíria para rapazes ou homens)) [...] ou com várias meninas [...] está sendo exposto [...]passar a noite toda transando com várias mulheres ou uma mulher pode passar a noite toda transando com vários homens ....é isso... (E1-entrevista individual).

Quanto aos fatores que influenciam para ter relações sexuais e não usar o preservativo, os adolescentes referem que o uso de álcool e outras drogas representam um risco para contrair HIV/ Aids, diante da quantidade de efeitos secundários que essas substâncias provocam.

[...] muitas vezes, os jovens... têm relações sexuais e podem além de ter relações sexuais usar drogas... a droga junto com a relação sexual... e isso também permite muitas doenças[...] (E1-entrevista individual).

[...] ]estariam em riscos de contrair uma doença de transmissão sexual bebendo muito. (E3-grupo focal)

[...]eu estaria exposto quando bebo muito... (E6-entrevista individual).

Os participantes apresentam o uso de drogas como um problema que pode originar consequências e ocasionar riscos para a saúde, incluindo o HIV.

\section{Dos riscos de contrair HIV/Aids nas relações sexuais às vulnerabilidades}

A categoria aborda que os encontros consecutivos no grupo focal permitiram aprofundar a compreensão sobre as representações sociais dos adolescentes, revelando um aumento da importância de dimensões psicossociais que expressam 
uma noção de passagem da preocupação com os riscos de contrair HIV/Aids nas relações sexuais para as representações de vulnerabilidades em saúde, segundo os adolescentes que participaram do estudo. Como apresenta a Figura 2.

Os adolescentes que participaram do estudo apresentam que outros elementos e aspectos da vida, para além dos biológicos, possuem relevância quando o tema é a sexualidade. Desse modo, mesmo sem a evocação do termo vulnerabilidade em saúde, o seu conceito pode ser compreendido nas falas dos estudantes, quando afirma envolvimentos individuais, como os comportamentos e capacidade de apreensão de informações disponíveis sobre o HIV; falta de conversas com os pais e familiares; e sociais como os relacionados à pobreza e a problemas econômicos, bem como dificuldades de acesso aos serviços de saúde e de educação em saúde na escola.

Sobre as dificuldades de acesso aos serviços de saúde, as falas abordam da ausência de conhecimentos sobre os atendimentos na rede de cuidados para o HIV, destacando dos serviços de testagem à ciência da gratuidade dos tratamentos. Os participantes destacam a ideia de que há muitas dificuldades para as pessoas que estão vivendo com HIV (PVHIV) tenham acesso ao sistema único de saúde (SUS) no Brasil.

\section{[...]muitos jovens não têm o conhecimento do como fazer e como não fazer ((sexo seguro)) ... acabam transmitindo a doença e não sabendo como fazer os exames para poder se prevenir... para poder se cuidar... até passando para outros jovens[...] (E2-grupo focal). \\ [...] para tratar ela ((a Aids)) é difícil porque o sistema de saúde... apesar de estar sendo ótimo... a importância do SUS... ainda é bem precário... então para se tratar esse negócio ((a Aids)) eu acho muito difícil [...] (E8- grupo focal).}

Nas falas dos participantes, outros destaques atrelados às dificuldades de acesso aos serviços de saúde são as dificuldades para efetivar a educação sexual na escola que vão desde aspectos políticos-ideológicos, religiosos à imaturidade para tratar destes temas de maneira séria nos diversos ambientes, incluindo o escolar.

[...]uma coisa importante é discutir sobre a relação sexual na escola... porque muitas ((meninas)) estão no primeiro ano e já tem relações sexuais.... mas não sabem os métodos preventivos [...]não vão ao ginecologista ou nunca foram.... não sabem que têm que fazer um exame por ano ((refere-se ao exame preventivo para o câncer do colo do útero)) [...]e outras coisas[...]o Estado não está querendo que aprendam disso... eles não querem que estudemos gênero... imagina educação sexual... (E4-grupo focal).

[...]eles acham que se o professor .... explicar sobre sexo.... sobre doenças.... sobre gênero que é um assunto que nós devemos saber.... estão nos influenciando[...] tem.... na escola... o ((Programa Saúde na Escola)) educação na escola... mas não é tão informativo.... vivemos em uma sociedade que é fundamentalmente religiosa... umas não tanto quanto outras... mas a nossa sociedade é religiosa[...] mas nós sentimos muito o peso da religião... então eu acho que se influencia bastante em relação ao tabu[...] (E3-grupo focal).

[...]os adolescentes vêm apresentando um desenvolvimento sexual muito rápido muito cedo e nas escolas não falam.... tipo quando estão na sétima ou oitava série já tem meninas que transam e ninguém fala porque é um assunto tabu.... tanto que para se apresentar um trabalho sobre DST ((doenças sexualmente transmissíveis)) no colégio ninguém tem maturidade para nada... era uma pessoa apresentando e os outros rindo... todo mundo rindo... a pessoa chegava com uma maquete e uma camisinha... e o pessoal faz.... todo mundo sabe todo mundo conhece... mas pelo assunto ser tão polêmico.... todo mundo fica meio acuado... (E2-grupo focal). 
O tema ter conversas com os pais ou familiares, ganhou destaque nas falas dos adolescentes, sendo o relacionamento familiar comprometido apresentado por eles como responsável pelo desconhecimento ou não adoção da prevenção.

[...]eu mesma não conversava com meus pais... eles não sentaram e disseram vamos conversar... não sentaram... eu fiquei sabendo por um e por outro[...] a confiança que tem em casa e, muitas vezes, quando os pais deixam assim como tanto faz... a menina vai pelo lado errado... o menino vai pelo lado errado... vai porque o que se escuta na rua... no colégio é vai menina... vai menino... é sempre assim nunca vão dizer menina pensa direitinho no que está fazendo... não é assim... não... é sempre tu não queres? Então, vai!... depois quando vai cair na real ((gíria para enfrentar a realidade)) já foi... uma gravidez... uma doença. (E3-grupo focal).

O comprometimento da participação familiar ou a falta de conversas com os pais ainda é mais crítico para as adolescentes femininas. Todas as participantes relataram que os pais não costumam conversar sobre sexo e questões pertinentes à prevenção da gravidez e de doenças sexualmente transmissíveis, como apresentado nas falas a seguir.

[...] o meu caso, por exemplo, eu tenho dois irmãos homens e eu de mulher... então, minha mãe chega, se senta na mesa e conversa com meus dois irmãos [...] ela me manda fechar a porta... ((relata que a mãe diz)) "- Tu não podes escutar... porque tu és mulher!" [...] (E1-grupo focal).

[...]ela ((a mãe)) nunca se sentou comigo [...] para dizer: ter relação sexual é isso, isso e isso... e eu acabei descobrindo as coisas... mas com meu irmão ela chegou e disse... camisinha é blá, blá, se coloca desta forma... mas eu aprendi sozinha... e quando ela descobriu que eu tinha perdido a virgindade ela ((relata que a mãe disse)) "- Ah... você precisa se casar" [...] (E3-grupo focal).

[...]toda semana ela ((a mãe de adolescente masculino)) me dava umas três ou quatro camisinhas... sem brincadeira! (E7-grupo focal).

Quanto a presença do tema ser pobre, avultasse nos resultados que aparece de modo controverso. Os adolescentes do estudo, embora moradores de periferias do município, atribuem a jovens pobres o maior número de casos de doenças sexualmente transmissíveis. No entanto, eles não revelam percepção social sobre essa vulnerabilidade em saúde para si.

[...]pode parecer um pouco preconceituoso, mas normalmente sempre é a camada mais pobre que contêm mais essas doenças... (E4-grupo focal).
[...] não sendo preconceituoso... também eu acho que vocês também são preconceituosos... mas... as favelas e as periferias... que não aprende isso com facilidades ((refere-se à proteção e prevenção)) (E3-grupo focal).

Os adolescentes referem que o fato de ser pobre pode levar outros adolescentes a terem relações sexuais sem o uso do preservativo, mesmo considerando que essas afirmações podem ser preconceituosas.

\section{DISCUSSÃO}

As representações sociais de adolescentes sobre os riscos para contrair o HIV/Aids nas relações sexuais remetem a representações e conteúdos representacionais como não usar preservativo, ter usado álcool e/ou drogas e ter vários parceiros, apresentados como resultados na primeira categoria.

Quanto a não usar preservativo, o estudo compreendeu que a representação envolve dimensões emocionais, afetivas e sociais como a confiança no parceiro e a demonstração desse sentimento amoroso ou romântico na negociação do uso com os parceiros. Por isso, relataram dispensar o uso do preservativo nas relações sexuais com parceiros fixos, namorados e namoradas, mesmo conhecendo a importância do seu uso, como também encontraram outros autores ${ }^{12}$.

No entanto, foi mais apresentado entre os adolescentes masculinos que o motivo para o não uso é a maior prioridade em ter relações sexuais no momento imediato, sem pensar nas consequências, já que a intensidade e o prazer sexual representam suas maiores metas idealizadas, corroborando com os achados de um estudo realizado com alunos do ensino médio de uma escola pública de Fortaleza, Ceará, Brasil ${ }^{13}$.

Quanto a ter vários parceiros, as representações remetem à promiscuidade e a vivência desviante da sexualidade, consolidando ideais normativos sobre o sexo, presentes em suas realidades, bem com a necessidade de afirmação grupal no processo de desenvolvimento da sexualidade e no contexto biopsicossocial ${ }^{13}$. Nesse contexto, deve-se atentar para o fato de que o tema não remete ao não uso do preservativo com esses vários parceiros, mas, sim, atribui o risco ao fato de se possuir muitos parceiros ou ter muitas relações sexuais na mesma noite.

Esses achados são muito preocupantes e revelam a necessidade de discutir os fatores associados ao uso de preservativo entre adolescentes para o planejamento de intervenções. Sobre isso, um inquérito de base populacional revelou que entre àqueles que iniciaram a atividade sexual após os 15 anos; que usaram preservativo na primeira relação sexual; e que têm acesso a preservativos gratuitos o uso foi mais frequente $e^{14}$.

Quanto a ter usado álcool e/ou drogas como um dos riscos para contrair o HIV/Aids, os adolescentes participantes do presente estudo apresentam que a compreensão das representações sociais dos adolescentes sobre a questão das bebidas alcoólicas e, mais precisamente, sobre o alcoolismo favorece conhecer o entendimento que eles têm sobre esse objeto psicossocial no 
seu cotidiano, ${ }^{15} \mathrm{e}$, por sua vez, como influenciam suas práticas e escolhas sobre o uso do preservativo nas relações sexuais.

No Brasil, um estudo de revisão que reuniu pesquisas sobre os fatores associados e os comportamentos sexuais que contribuem para doenças sexualmente transmissíveis entre adolescentes, identificou que o uso de substâncias como álcool e drogas aumentam a incidência de relações sexuais desprotegidas ${ }^{16}$.

$O$ achado se repete no contexto internacional, um estudo sobre o comportamento de risco relacionado ao uso de preservativos e anticoncepcionais entre estudantes sexualmente ativos do ensino médio nos Estados Unidos (EUA) demonstrou que a prevalência do uso de preservativo foi consistentemente menor entre os alunos que consumiram drogas e outras substâncias psicoativas como o álcool ${ }^{17}$.

Salienta-se que as mudanças nas compreensões dos riscos de contrair HIV/Aids nas relações sexuais às representações das vulnerabilidades foram sendo apresentadas pelos participantes do estudo, à medida que os encontros promovidos para os grupos focais permitiram aprofundar os dados das entrevistas individuais dos encontros iniciais.

Desse modo, a segunda categoria, denominada: dos riscos de contrair HIV/Aids nas relações sexuais às vulnerabilidades, que envolvem ter dificuldades de acesso aos serviços de saúde e de educação sexual nas escolas; não ter conversas com os pais ou familiares; e ser pobre, apresentadas como temas nos resultados.

Uma das principais e mais marcantes mudanças do perfil epidemiológico da doença iniciou o processo de transformação do uso do conceito de grupo de risco, depois de comportamentos de risco para o de vulnerabilidades. ${ }^{4}$ Os conhecimentos sobre os elementos críticos das vulnerabilidades para o HIV são gênero, classe social e faixa etária. Eles admitem a afirmação de que o vírus é um fenômeno social ligado a vivência da sexualidade humana ${ }^{18-19}$.

Há três dimensões complexas e interdependentes na noção das vulnerabilidades: programática ou institucional, social e individual. Essa última subdivide-se em dois níveis, um cognitivo e outro comportamental, o que pode ser relacionado aos resultados no presente estudo, às dificuldades de transformar os conhecimentos sobre os riscos em atitudes de prevenção ${ }^{2}$. As dimensões programáticas e sociais das vulnerabilidades também foram abordadas pelos participantes do estudo, quando apresentaram as dificuldades para o acesso aos serviços de saúde para testagens, aconselhamento e distribuição de insumos e tratamentos e para a educação sexual nas escolas.

Quanto à educação sexual nas escolas, o Brasil ratificou que parcerias com escolas e comunidades deveriam ser realizadas pela Estratégia de Saúde da Família (ESF) para diminuir vulnerabilidades entre adolescentes. No entanto, aspectos políticos-ideológicos têm comprometido sua efetivação ao longo dos anos ${ }^{20}$, e isso também foi referido pelos adolescentes no estudo.

Salienta-se que pesquisa realizada com estudantes na Etiópia considerou altamente recomendado incluir a saúde sexual e reprodutiva no currículo educacional ${ }^{21}$. Corroborando com os achados do presente estudo, quando aponta que os adolescentes aprendem sobre as vulnerabilidades e os riscos entre si mais do que no núcleo familiar ou com os professores. Assim, sobre o tema, alguns pesquisadores descrevem que as estratégias que promovam o protagonismo dos próprios estudantes no processo educativo, como no uso de metodologias ativas e de educação por pares são mais efetivas ${ }^{22-23}$.

Vale destacar que essa noção das vulnerabilidades permite a proposição de que políticas públicas podem impactar a prevenção do HIV, tendo em vista que apenas o conhecimento dos riscos não implica em mudanças de atitude e comportamentos, diante dos muitos fatores biopsicossociais que influenciam, como apontaram os achados de um estudo longitudinal realizado no Zimbábue $^{24}$

Outros estudos consideram crucial envolver os pais, familiares e escolas nas ações de promoção e prevenção, tendo em vista que muitas orientações inadequadas de amigos e de fontes não confiáveis são recebidas pelos adolescentes ${ }^{20,22}$. O que corrobora com os achados desse estudo frente a ausência de conversas com os pais ou familiares sobre o tema. Sobre isto, a vulnerabilidade social expressa por um relacionamento familiar comprometido foi ainda mais intensa entre as adolescentes femininas.

De outra vulnerabilidade social, compreendida a partir das falas dos adolescentes que participaram do estudo, emergiram representações ancoradas por eles com relação à pobreza (ser pobre). Os adolescentes representam a mesma como um fator relacionado à contaminação ou contágio pelo HIV, já que, segundo a perspectiva dos participantes, as pessoas pobres não têm educação adequada ou recursos econômicos suficientes para proteção e, por isso, seria o grupo mais susceptível ao HIV ou outras Infecções Sexualmente Transmissíveis (IST).

Contudo, mesmo enquadrando-se nesse grupo social em relação aos aspectos econômicos investigados, ainda não representam a si mesmos como vulneráveis porque não se reconhecem assim ${ }^{25}$.

\section{CONSIDERAÇÕES FINAIS E IMPLICAÇÕES PARA A PRÁTICA}

O estudo permitiu compreender que as representações sociais de adolescentes sobre vulnerabilidades e riscos para contrair o HIV/Aids nas relações sexuais estão relacionadas ao não usar preservativo, por questões como o prazer sexual para os adolescentes masculinos e às dificuldades femininas para levá-lo consigo.

Ter usado álcool e/ou drogas, ter vários parceiros, ter dificuldades de acesso aos serviços de saúde e de educação sexual nas escolas; e não ter conversas com os pais ou familiares, este último mais crítico para adolescentes femininas, são outras vulnerabilidades e riscos que emergiram no estudo.

Destaca-se que a compreensão sobre as representações sociais dos adolescentes revelou um aumento da importância de dimensões psicoemocionais e sociais que expressam uma 
noção de passagem da preocupação com os riscos de contrair HIV/Aids nas relações sexuais para as representações de vulnerabilidades em saúde.

Os resultados deste estudo propõem como implicação prática a priorização de políticas públicas focadas nas dimensões emocionais, afetivas e sociais no ambiente em que as representações circulam, considerando-se que nesse cenário podem ser capazes de interferir na realidade dos adolescentes.

Quanto às limitações do estudo, considerou-se as relacionadas ao tamanho da amostra, que de 15 participantes no período das entrevistas individuais foi resumido a oito nos encontros do grupo focal, em virtude da disponibilidade dos estudantes, considerando tratar-se de uma escola de ensino integral. No entanto, para minimizar os efeitos dessa limitação, realizou-se uma extensão do período de coleta dos dados e agendamento prévio das participações de todos os alunos, a fim de prevenir perdas de novos participantes a cada encontro. O que tornou possível manter a participação dos mesmos oito estudantes para todos os encontros subsequentes.

\section{FINANCIAMENTO}

O presente trabalho foi realizado com apoio da Coordenação de Aperfeiçoamento de Pessoal de Nível Superior - Brasil (CAPES) - Código de Financiamento 001, na modalidade bolsa de Mestrado concedida a Esmelsy Cabrera Garcia, no período de 2015 à 2017.

\section{CONTRIBUIÇÕES DOS AUTORES}

Desenho do estudo. Esmelsy Cabrera Garcia. Regina Celia de Oliveira. Carlos Roberto Lyra da Silva. Amanda Regina da Silva Góis. Fátima Maria da Silva Abrão.

Coleta ou produção dos dados. Esmelsy Cabrera Garcia. Ivoneide Ribeiro Costa.

Análise de dados. Esmelsy Cabrera Garcia. Amanda Regina da Silva Góis. Fátima Maria da Silva Abrão.

Interpretação dos resultados. Esmelsy Cabrera Garcia. Ivoneide Ribeiro Costa. Regina Celia de Oliveira. Carlos Roberto Lyra da Silva. Amanda Regina da Silva Góis. Fátima Maria da Silva Abrão.

Redação e revisão crítica do manuscrito. Esmelsy Cabrera Garcia. Ivoneide Ribeiro Costa. Regina Celia de Oliveira. Carlos Roberto Lyra da Silva. Amanda Regina da Silva Góis. Fátima Maria da Silva Abrão.

Aprovação da versão final do artigo. Esmelsy Cabrera Garcia. Ivoneide Ribeiro Costa. Regina Celia de Oliveira. Carlos Roberto Lyra da Silva. Amanda Regina da Silva Góis. Fátima Maria da Silva Abrão.

Responsabilidade por todos os aspectos do conteúdo e a integridade do artigo publicado. Esmelsy Cabrera Garcia. Ivoneide Ribeiro Costa. Regina Celia de Oliveira. Carlos Roberto Lyra da Silva. Amanda Regina da Silva Góis. Fátima Maria da Silva Abrão.

\section{EDITOR ASSOCIADO}

Aline Cavicchioli Okido (B)

\section{EDITOR CIENTÍFICO}

\author{
Ivone Evangelista Cabral (D)
}

\section{REFERÊNCIAS}

1. Brasil. Ministério da Saúde. Boletim Epidemiológico HIV e Aids-2018 [Internet]. 2018 [citado fev 01 2021]. Disponível em: http://www.aids. gov.br/pt-br/pub/2018/boletim-epidemiologico-hivaids-2018.

2. Ayres JR, Castellanos MEP, Baptista TWF. Entrevista com José Ricardo Ayres. Saude Soc. 2018 jan;27(1):51-60. http://dx.doi.org/10.1590/ s0104-12902018000002.

3. Fontes MB, Crivelaro RC, Scartezini AM, Lima DD, Garcia AA, Fujioka RT. Determinant factors of knowledge, attitudes and practices regarding STD/AIDS and viral hepatitis among youths aged 18 to 29 years in Brazil. Cien Saude Colet. 2017 abr;22(4):1343-52. http://dx.doi. org/10.1590/1413-81232017224.12852015. PMid:28444057.

4. Calazans GJ, Pinheiro TF, Ayres JRCM. Vulnerabilidade programática e cuidado público: Panorama das políticas de prevenção do HIV e da Aids voltadas para gays e outros HSH no Brasil. Sex Salud Soc. 2018;(29):263-93. http://dx.doi.org/10.1590/1984-6487.sess.2018.29.13.a.

5. Kinalski DDF, Kleinubing RE, Bick MA, Langendorf TF, Padoin SMM, Cardoso PC. Line of care for children and teenagers living with HIV: participatory research with professionals and managers. Esc Anna Nery. 2021;25(2):e20200266. http://dx.doi.org/10.1590/2177-9465ean-2020-0266.

6. Silva AA, Gubert FA, Barbosa-Filho VC, Freitas RWJF, Vieira-Meyer APGF, Pinheiro MTM et al. Ações de promoção da saúde no Programa Saúde na Escola no Ceará: contribuições da enfermagem. Rev Bras Enferm. 2021;74(1):e20190769. http://dx.doi.org/10.1590/0034-71672019-0769. PMid:33787775

7. Jodelet D. O movimento de retorno ao sujeito e a abordagem das Representações Sociais. Sociedade e Estado, Brasília. 2009 set dez;24(3):679-712. http://dx.doi.org/10.1590/S0102-69922009000300004

8. Lei 8.069, de 13 de julho de 1990 (BR). Dispõe sobre o Estatuto da Criança e do Adolescente e dá outras providências. Diário Oficial da União [periódico na internet], Brasília (DF), 1990 [citado fev 01 2021]. Disponível em: http://www.planalto.gov.br/ccivil_03/leis//8069.htm

9. Oliveira DC. Análise de conteúdo temático-categorial: uma técnica maior nas pesquisas qualitativas. In: Lacerda MR, Costenaro RGS, editores. Metodologia da pesquisa para a Enfermagem e Saúde: da teoria à prática. Porto Alegre: Moriá; 2016. p. 481-511.

10. Medeiros HP, Teixeira E. Metodologia da pesquisa para a enfermagem e saúde: resenha de livro. Rev Bras Enferm. 2016;69(5):1000-1. http:// dx.doi.org/10.1590/0034-7167-2015-0135.

11. Jodelet $\mathrm{D}$. Ponto de vista: sobre o movimento das representações sociais na comunidade científica brasileira. Temas Psicol. 2011;19(1):19-26.

12. Correa LDR, Gonzaga LL. Rejeição do uso de camisinha por adolescentes: uma perspectiva a partir da zona muda das representações sociais. Rev Espaço Pedagógico. 2018;25(2):472-87.

13. Bezerra EO, Pereira MLD, Chaves ACP, Monteiro PV. Social representations of teenagers on sexual relations and the use of condoms. Rev Gaúcha Enferm. 2015 mar;36(1):84-91. http://dx.doi.org/10.1590/19831447.2015.01.45639. PMid:26098807.

14. Gutierrez EB, Pinto VM, Basso CR, Spiassi AL, Lopes MEBR, Barros CRDS. Fatores associados ao uso de preservativoem jovens - inquérito de base populacional. Rev Bras Epidemiol. 2019;22:e190034. http:// dx.doi.org/10.1590/1980-549720190034. PMid:31038615.

15. Pereira ASK, Medeiros ED, Fernandes AL, Pereira BR. Representações sociais do álcool entre estudantes universitárias brasileiras. Psicol Conoc Soc. 2019 jun;9(1):161-81.

16. Moura LR, Torres LM, Cadete MMM, Cunha CF. Factors associated with health risk behaviors among Brazilian teenagers: an integrative review. Rev Esc Enferm USP. 2018;52:e03304. PMid:29668787.

17. Szucs LE, Lowry R, Fasula AM, Pampati S, Copen CE, Hussaini KS et al Condom and contraceptive use among sexually active high school students - youth risk behavior survey, United States, 2019. MMWR 
Suppl. 2020;69(1):11-8. http://dx.doi.org/10.15585/mmwr.su6901a2. PMid:32817600.

18. Souza SO, Cunha PA, Almeida SC, Ribeiro SCP, Marcelly SMM, Matos MM Iniquidades de género e vulnerabilidade às IST/HIV/AIDS em adolescentes de assentamento urbano: um estudo exploratório. Cienc Enferm. 2020;26:12.

19. Góis ARS, Oliveira DC, Costa SFG, Oliveira RC, Abrão FMS Representações sociais de profissionais da saúde sobre as pessoas vivendo com HIV/Aids. Av en Enfermería. 2017;35(2):169-78. http:// dx.doi.org/10.15446/av.enferm.v35n2.59636.

20. Moreira P, Reis T, Mendes R, Menezes A. HIV vulnerability among teenagers attending to public schools. RPCFO. 2019 jul;11(4):868-72. http://dx.doi.org/10.9789/2175-5361.2019.v11i4.868-872.

21. Nigussie T, Yosef T. Knowledge of sexually transmitted infections and its associated factors among polytechnic college students in Southwest Ethiopia. Pan Afr Med J. 2020;37:68. http://dx.doi.org/10.11604/ pamj.2020.37.68.22718. PMid:33244331.
22. Barreto VP, Inácio JO, Silva BCO, Aquino ARG, Marques CC, Feijão AR. Peer education as strategy for HIV/AIDS prevention among teenagers. Saúde Pesqui. 2020;13(2):253-63. http://dx.doi.org/10.17765/21769206.2020v13n2p253-263.

23. Carvalho ACT, Silva DMR, Brandão-Neto W, Pereira EBF, Menezes MLN, Aquino JM. Percepções de adolescentes escolares do sexo masculino quanto ao cuidado à sua saúde. Enferm Actual Costa Rica. 2019 dez;(37):80-94.

24. Schaefer R, Thomas R, Maswera R, Kadzura N, Nyamukapa C, Gregson $\mathrm{S}$. Relationships between changes in HIV risk perception and condom use in East Zimbabwe 2003-2013: population-based longitudinal analyses. BMC Public Health. 2020;20(1):756. http://dx.doi.org/10.1186/ s12889-020-08815-1. PMid:32448365.

25. Herzog L. Teoria do reconhecimento e democracia econômica: potenciais não exauridos. Civitas. 2018;18(3):523-38. http://dx.doi. org/10.15448/1984-7289.2018.3.29550. 